\title{
Magnetic resonance-guided laser interstitial thermal therapy for pediatric periventricular nodular heterotopia-related epilepsy
}

\author{
Vijay M. Ravindra, MD, MSPH, ${ }^{1,2}$ Sungho Lee, MD, PhD, ${ }^{3}$ David Gonda, MD, ${ }^{4}$ Ilana Patino, PA-C, ${ }^{1}$ \\ Lucia Ruggieri, PA-C, ${ }^{1}$ Daniel S. Ikeda, MD, ${ }^{5}$ and Daniel J. Curry, MD ${ }^{1,3}$ \\ 'Division of Pediatric Neurosurgery, Texas Children's Hospital, Baylor College of Medicine, Houston; and ${ }^{3}$ Department of \\ Neurosurgery, Baylor College of Medicine, Houston, Texas; 'Department of Neurosurgery, Clinical Neurosciences Center, \\ University of Utah, Salt Lake City, Utah; " ${ }^{2}$ eppartment of Neurosurgery, Rady Children's Hospital, University of California, San \\ Diego, California; and ${ }^{5}$ Department of Neurosurgery, US Naval Hospital Okinawa, Okinawa, Japan
}

OBJECTIVE Periventricular nodular heterotopia (PVNH) is a result of disrupted neuronal migration from the ventricular system and can be a rare cause of refractory focal epilepsy. The goal of this case series was to describe the treatment of pediatric PVNH-related epilepsy with MR-guided laser interstitial thermal ablation.

METHODS Patients treated at a single institution with MR-guided laser interstitial thermal therapy (MRgLITT) for PVNH-related epilepsy were identified. Preoperative and postoperative seizure outcomes and procedural information were evaluated.

RESULTS Five children with PVNH treated with MRgLITT were reviewed; 1 child was treated twice. Three patients were female; the median age was 10.9 years. Five of 6 treatments were preceded by stereoelectroencephalography phase II monitoring. Three children experienced unilateral PVNH, and 2 had bilateral seizures. The median number of seizures recorded during phase II monitoring was 2; the median number of ablation targets was 2 (range 1-4). All patients experienced a decrease in seizure frequency; 4 patients (80\%) had an Engel class $\leq \mathrm{III}$ at the last follow-up (range I-IV). One child experienced right hemianopia posttreatment.

CONCLUSIONS This case series investigation has illustrated a novel, minimally invasive approach for treating pediatric PVNH-related epilepsy. Further study of this technique with comparison with other surgical techniques is warranted.

https://thejns.org/doi/abs/10.3171/2021.5.PEDS21171

KEYWORDS periventricular nodular heterotopia; stereoelectroencephalography; laser ablation; electrocorticography; medically refractory epilepsy; magnetic resonance-guided laser interstitial thermal therapy; surgical technique

$\mathrm{M}$ EDICALLY refractory epilepsy is a devastating condition that affects millions of people worldwide. ${ }^{1}$ In children, the effects of untreated or poorly managed epilepsy can have a profound impact on development, behavior, and speech. Periventricular nodular heterotopia (PVNH) is a rare and difficult-to-treat cause of medically refractory epilepsy that lacks a widely adopted treatment strategy. ${ }^{2}$

PVNH is a disruption of outward neuronal migration from the ventricular system that results in the formation of ectopic nodules yielding heterotopic gray matter located in the periventricular region. ${ }^{3,4}$ What role heterotopic gray matter plays in seizure production is poorly understood. It is postulated that complex interactions between the het- erotopic nodules and the allo- or neocortex are present in patients with PVNH and epilepsy. ${ }^{5}$ There is distinct heterogeneity within PVNH. The nodules can vary from a single distinct nodule to multiple nodules distributed ubiquitously along the bilateral ventricles. ${ }^{3}$ The concern comes from precise localization of a seizure focus. In addition, PVNH can be associated with other epilepsy generators including hippocampal sclerosis, polymicrogyria, and focal cortical dysplasia. ${ }^{6-8}$ The surgical treatment of PVNHrelated epilepsy is difficult because the deep periventricular location and the overlying cortex increase the potential risk of significant neurological deficits. ${ }^{9}$

MR-guided laser interstitial thermal therapy (MRgLITT) is a well-established technique in neurosurgery, specifically

ABBREVIATIONS AED = antiepileptic drug; MEG = magnetoencephalography; MRgLITT = MR-guided laser interstitial thermal therapy; PVNH = periventricular nodular heterotopia; RNS = responsive neurostimulation; SEEG = stereoelectroencephalography.

SUBMITTED March 31, 2021. ACCEPTED May 12, 2021.

INCLUDE WHEN CITING Published online September 24, 2021; DOI: 10.3171/2021.5.PEDS21171. 
in epilepsy surgery ${ }^{10,11}$ that avoids a large open surgery while enabling focal treatment of epileptogenic lesions. ${ }^{11}$ With respect to treating PVNH, the heterotopic nodules can be precisely ablated while the overlying cortex, which is often eloquent, ${ }^{3}$ is preserved. It can also allow for inclusion of the cortex in the therapy, skipping the white matter tracts, when it is noneloquent. Recent reports have suggested the use of laser ablation for PVNH-related epilepsy, ${ }^{2,9}$ but large reports are lacking in children.

We aimed to describe the use of MRgLITT in pediatric PVNH-related epilepsy and to report preliminary outcomes with the goal of illustrating the potential safety and efficacy of the technique.

\section{Methods}

\section{Study Design and Data Collection}

This was a single-center retrospective study of consecutively treated pediatric patients ( $<18$ years of age) treated with MRgLITT for PVNH-related epilepsy at Texas Children's Hospital from January 1, 2014, through December 31, 2019, by the senior author (D.J.C.). IRB approval was obtained with a waiver of informed consent.

\section{Clinical Variables}

Demographic information, including age, sex, weekly seizure frequency, number of preoperative antiepileptic drugs (AEDs), total number of AEDs trialed, number of postoperative medications, and follow-up duration, was collected. Surgical variables recorded included method of phase II monitoring, duration of phase II monitoring, number and type of seizures experienced during phase II monitoring, number of ablation targets, length of hospital stay, neurological complications, and other sources of potential morbidity.

\section{Seizure Outcomes}

The primary outcome was Engel classification at the last follow-up..$^{12}$ The presence of long-term neurological complications, notably visual field deficits, motor deficits, and speech deficits, was also recorded.

\section{Patient Selection}

All patients were evaluated and treated at the Texas Children's Hospital Comprehensive Epilepsy Center. Children who were recommended for treatment of PVNH-related epilepsy were evaluated by a pediatric epileptologist and confirmed to have a diagnosis of medically refractory epilepsy (defined as uncontrolled epilepsy after being trialed on three or more AEDs). All children were discussed in a weekly multidisciplinary conference, including neurologists, neurosurgeons, social workers, neuropsychologists, and radiologists, regarding potential surgical patients. All patients underwent presurgical evaluation with video-EEG recording as well as neuropsychological testing (when capable), and CT and MRI. Some patients also received additional ictal/interictal SPECT; PET and magnetoencephalography (MEG) were used on a case-bycase basis, depending on presentation and semiology. All decision-making for invasive monitoring was made based on phase I information, including semiology, anatomical abnormality on MRI, video-EEG findings with electrical brain analysis, and MEG, PET, and SPECT findings. Patients with concordant data suggestive of a lateralizing lesion were recommended to proceed with MRgLITT. If the data were suggestive but further confirmation was necessary, phase II monitoring was recommended, followed by therapeutic treatment, if appropriate.

\section{Surgical Treatment}

If phase II monitoring was recommended, stereoelectroencephalography (SEEG) using robot-assisted stereotactic placement was performed to locate the seizure onset zone. ${ }^{13-18}$ The technique for MRgLITT has been previously published in detail. ${ }^{10,11}$ Ablation is performed under general anesthesia with the patient in rigid cranial fixation. If a new trajectory is desired, a small stab incision is made after registration with the robotic stereotactic system. An anchor bolt is then placed, and the laser catheter is placed into the seizure onset zone. If the trajectory is the same as an SEEG electrode, then a sterile exchange is performed for the same trajectory. The laser probe is passed through the skull bolt to its target. The ablation is performed using the Visualase Thermal Therapy System (Medtronic, Inc.), which uses a 15-W generator, a 980-nm-diode laser, and a $1.65-\mathrm{mm}$-diameter applicator. The connected computer workstation processes the images in real time.

The patient is transferred into the MRI magnet, and the ablation system is activated to thermally ablate the target tissue and any surrounding heterotopic tissue. Heating is monitored by phase difference imaging with gradientecho pulse sequence acquisition. The treatment workstation creates a color-coded temperature and predicted tissue damage map (irreversible damage estimate) that is displayed on the monitor in real time. If the focus of the ablation is both the nodule and the overlying cortex, the ablation is performed on the nodule first, with low-limit markers placed on the surrounding white matter with the threshold lowered to $48^{\circ} \mathrm{C}$. The laser is then withdrawn from within the catheter to center the heat on the cortex of the trajectory. When adequate ablation is achieved, the patient is removed from the MR scanner, the laser is removed, and a single skin stitch is placed for closure. Figure 1 shows a preoperative T1-weighted axial MR image demonstrating right-sided PVNH in patient 1 . Figure 2 is a diffusion-weighted MR sequence for the same patient demonstrating successful ablation of the target with preservation of the superficial cortical architecture.

\section{Statistical Analysis}

Data were descriptively reported as means, median, and range for continuous variables and with counts and frequencies for categorical data.

\section{Results \\ Demographics}

Six laser ablations for PVNH-related epilepsy were performed in 5 children. One patient (patient 5) had a 40\% reduction in seizure frequency during an initial ablation and underwent retreatment with an additional episode of 

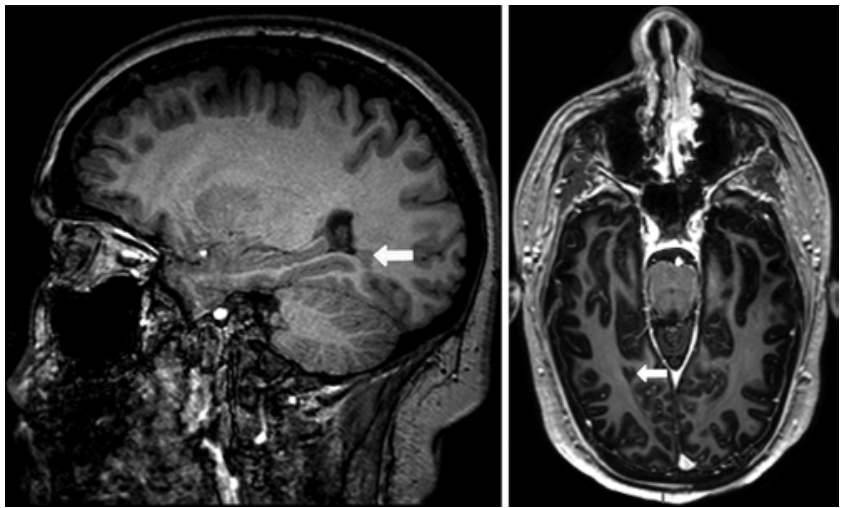

FIG. 1. Preoperative T1-weighted sagittal MR image (left) and T1weighted contrast-enhanced axial MR image (right) obtained in patient 1 demonstrating right-sided PVNH (arrows).

phase II monitoring followed by a second ablation of a different target. Table 1 outlines the baseline demographic information and disease characteristics of the 5 children receiving 6 treatments. The median patient age at the time of treatment was 10.9 years (range 5.3-18.4 years). Sixty percent of the children were female.

The mean number of seizures experienced weekly was 74.4 (median 35 [range 3-210]). All children had a diagnosis of PVNH, and none had a diagnosed genetic or syndromic condition. None of the children had a vagal nerve stimulator before treatment. Patient 5 had a responsive neurostimulation (RNS) device placed after the second ablation treatment to aid in seizure control when a second seizure onset zone was defined on SEEG in visually eloquent cortex. The mean number of preoperative AEDs was 3.4, with a median of 3 (range 2-5). The mean number of AEDs trialed was 9.2, with a median of 7 (range 6-13).

\section{Phase II Monitoring and Treatment Information}

Five of the 6 treatments were preceded by phase II monitoring with SEEG; patient 1 was directly treated with a single target and did not require phase II monitoring. The mean number of electrodes placed for phase II monitoring was 11 (median 11 [range 10-12]). The mean duration of phase II monitoring was 5.8 days (median 7 days [range 3-7 days]). The mean number of seizures experienced and recorded during phase II monitoring was 82 , with a median of 60 (range 3-180). Additionally, the median number of seizure types experienced during the phase II monitoring was 2 (range 1-5). A median of 2 (range 1-4) ablation targets were chosen for each child.

Three children had unilateral PVNH and were treated for singular, unilateral targets. Two children had bilateral PVNH (patients 4 and 5). Patient 4 was treated for a left-sided, unilateral target. Patient 5 underwent initial treatment on the right, with a $40 \%$ reduction in seizure frequency; 1.2 years later, the patient underwent left-sided treatment with concomitant RNS implantation.

\section{Seizure Outcomes}

The mean follow-up time for the cohort was $2.48 \pm 1.9$ years (median 2.4 years [range $0.37-5.36$ years]). The En-
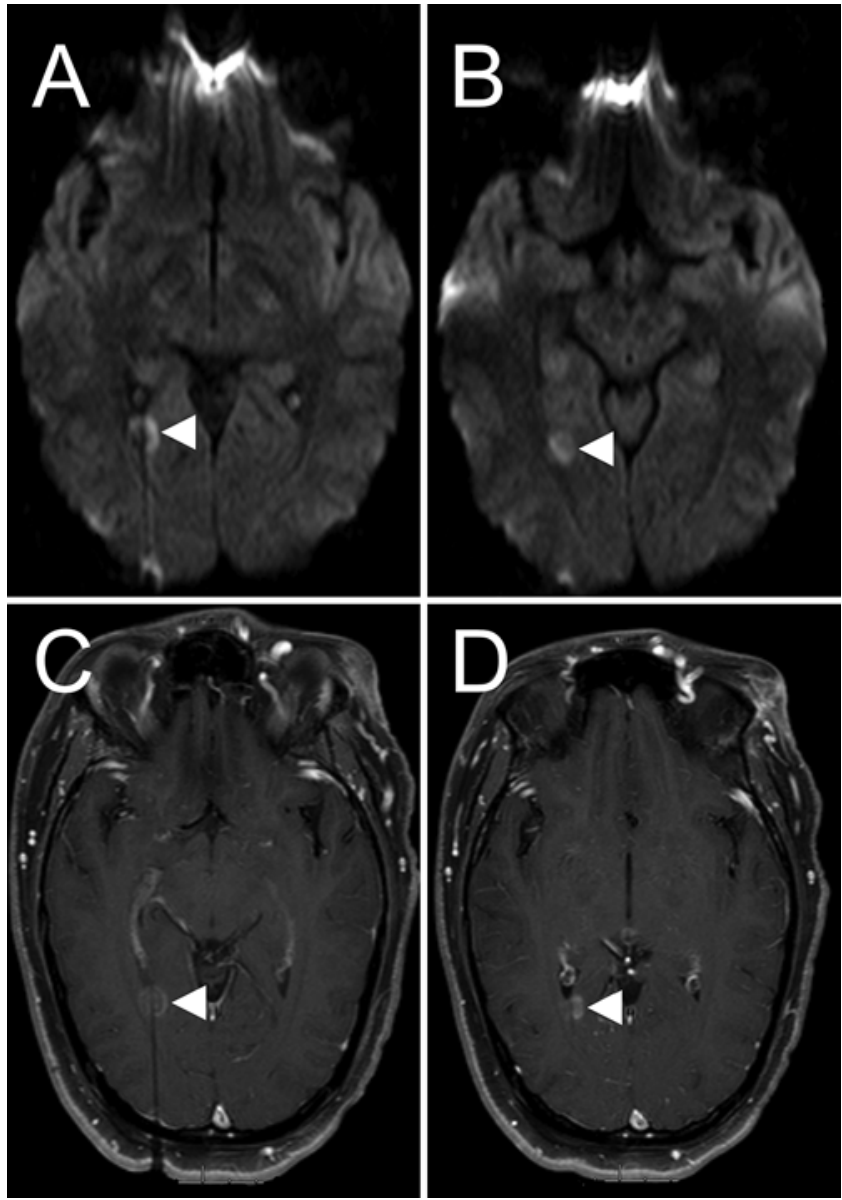

FIG. 2. Postoperative axial diffusion-weighted MR images (A and B) and axial T1-weighted contrast-enhanced slices ( $C$ and $\mathbf{D}$ ) obtained in patient 1 demonstrating ablation (arrowheads) of the previously demonstrated nodular heterotopia with preservation of the superficial cortex.

gel classification for each patient is presented in Table 1 . Overall, $80 \%$ of the children had an Engel class $\leq$ III at the final follow-up. The mean number of weekly seizures experienced postoperatively was $22.4 \pm 27.9$ (median 2 [range 0-70]). Two children had a reduction in seizure medication usage at the last follow-up; the other 3 children are on the same number of medications as they were preablation. The median number of days in the hospital after ablation was 3 (range 1-8 days).

\section{Complications}

None of the children who were treated experienced long-term motor or speech deficits. Based on formal visual field testing, 1 child (patient 4) experienced a right hemianopia posttreatment that had persisted at the latest follow-up. No patients experienced peri- or postoperative infections. No patient has died in the follow-up period.

\section{Discussion}

Treatment of PVNH-related epilepsy thus far has been complicated by our poor understanding of epileptogenesis, the presence of multifocal nodularity, and the deep loca- 
TABLE 1. Demographic and clinical data for patients with PVNH in this study

\begin{tabular}{|c|c|c|c|c|c|c|c|c|c|}
\hline Pt No. & $\begin{array}{l}\text { Age (yrs), } \\
\text { Sex }\end{array}$ & $\begin{array}{l}\text { Weekly Seizure } \\
\text { Frequency }\end{array}$ & $\begin{array}{l}\text { Phase II } \\
\text { Monitoring }\end{array}$ & Laterality & $\begin{array}{l}\text { No. of } \\
\text { Targets }\end{array}$ & $\begin{array}{l}\text { FU Duration } \\
\text { (days) }\end{array}$ & $\begin{array}{l}\text { Engel Class } \\
\text { at Last FU }\end{array}$ & $\begin{array}{c}\text { Weekly Seizure Frequency } \\
\text { After MRgLITT }\end{array}$ & Complications \\
\hline 1 & 15.6, M & 4 & NA & Unilat & 1 & 1960 & II & 2 & None \\
\hline 2 & 7.1, F & 210 & SEEG & Unilat & 2 & 134 & IV & 70 & None \\
\hline 3 & $5.3, \mathrm{~F}$ & 3 & SEEG & Unilat & 1 & 210 & III & 1 & None \\
\hline 4 & $10.9, \mathrm{~F}$ & 35 & SEEG & Bilat & 2 & 886 & I & 0 & Rt hemianopia \\
\hline $5^{*}$ & $\begin{array}{r}17.1, \mathrm{M} \\
18.4, \mathrm{M}\end{array}$ & $\begin{array}{r}120 \\
70\end{array}$ & $\begin{array}{l}\text { SEEG } \\
\text { SEEG }\end{array}$ & Bilat & $\begin{array}{l}4 \\
2\end{array}$ & $\begin{array}{r}875 \\
1340\end{array}$ & III & $\begin{array}{l}70 \\
39\end{array}$ & None \\
\hline
\end{tabular}

FU = follow-up; NA = not applicable; pt = patient.

* This patient was treated twice and had RNS device implantation concomitantly with the second ablation.

tion with overlying eloquent cortex and white matter. ${ }^{5} \mathrm{In}$ this paper, we describe the treatment of pediatric PVNHrelated epilepsy with MRgLITT to date.

Because of the difficult location and potential difficulty with localization in PVNH, minimally invasive monitoring with SEEG and subsequent MRgLITT represents a reasonable and feasible diagnostic and therapeutic option. The deep location of the PVNH makes localization with surface EEG alone challenging. Craniotomy and placement of subdural grid and strip electrodes not only increases the access-related morbidity but also frequently fails to locate the origin and spread of the deep seizure networks encountered in PVNH.

The use of MRgLITT allows for precise ablation of deep foci while preserving more superficial cortex; collateral damage to the adjacent white matter including the corticospinal and geniculocalcarine tracts is minimized. ${ }^{3}$ In contrast to open resection, MRgLITT has the advantage of improved selection of targeted tissue and minimization of destruction of eloquent cortex..$^{19}$ The same advantage holds when comparing MRgLITT with temporal lobectomy in the treatment of PVNH, specifically with regard to the risk of verbal memory deficit. ${ }^{20}$ Anterior temporal resection ${ }^{19,20}$ and radiofrequency ablation of $\mathrm{PVNH}^{21}$ have previously been described. Gamma Knife radiosurgery, although exhibiting a high safety profile by virtue of its noninvasiveness and low dosing, has a delayed efficacy in epilepsy of up to 3 years. ${ }^{19,20,22-24}$ Laser ablation allows for focal treatment of epileptogenic lesions while keeping in mind that multiple additional treatments may be needed in the future. ${ }^{11}$

Whiting et al. ${ }^{3}$ reported on 5 adults treated with 6 ablation procedures and demonstrated the safety of the technique. No patients in their series experienced postoperative dysphasia, hemiparesis, or infections; 1 patient experienced a postoperative quadrantanopia. The seizure outcomes were also favorable, with 3 patients who had an Engel class Ia outcome. The patients in their report, however, did not undergo phase II invasive intracranial monitoring. The authors suggested that for patients with a discrete periventricular nodular complex and concordant noninvasive data, as in their study population, proceeding directly to ablation is reasonable; ${ }^{3}$ however, in a more lesionally complex pediatric population, determination of the seizure onset zone may be more challenging. Only 1 child in our series (patient 1) was treated without phase II monitoring, and this child had a solitary nodule with concordant noninvasive data; this was the very first patient with PVNH to be treated using this technique. Scalp EEG demonstrated several seizure episodes arising from the right posterior occipital region. Additionally, the brain electrical source analysis suggested the nodule as the source, and the SPECT study demonstrated a minor increase in signal in the region of the posterior trigone periventricular heterotopia; thus, the decision was made to proceed directly with ablation. Although the patient did have a 50\% reduction in seizure burden after surgery, stage II monitoring data were deemed important for future cases. As a result of our iterative process improvement method, each patient thereafter with a suggestive nodule and PVNH underwent stage II monitoring.

Table 2 presents all reports of MRgLITT in the literature to date. , $3,9,21,25-27$ Thirteen patients with PVNH have been reported to have undergone MRgLITT alone, while 2 patients underwent both laser interstitial thermal therapy and anterior temporal lobectomy. In comparison with the previous reports in which 8 of $13(62 \%)$ adult patients had Engel class I outcomes, the overall outcomes in the current pediatric series are not as favorable; however, our outcomes were similar to those in the report in 2018 of Fayed et al., ${ }^{26}$ who reported on 3 pediatric patients who underwent MRgLITT for PVNH, with 2 patients having Engel class III outcomes and 1 patient having an Engel class I outcome. In our series, 2 patients had an Engel class III outcome, and 1 patient each had an Engel class I and II outcome. This is likely a reflection of more severe disease in pediatric patients with PVNH, who are likely to experience other brain anomalies as well. It is possible that, for children with bilateral PVNH, a stepwise, and potentially multimodal, approach may be necessary.

In this report, we describe ablation of the overlying cortex in noneloquent cases. The rationale for this practice comes from examination of PVNH as a migrational disorder, where the nodules represent deep areas of heterotopia that extend radially superficially. To improve seizure outcomes, this superficial area is targeted when dealing with noneloquent cortex. Localization of this with stage II monitoring is difficult, but the approach we have taken is to ablate the less obvious "cortical dysplasia" that is associated with the PVNH in an effort to be thorough and to maximize seizure freedom.

MRgLITT use may be accompanied by the need for tissue diagnosis; in these cases, biopsy is performed with 
TABLE 2. Reports of patients with PVNH treated with MRgLITT

\begin{tabular}{|c|c|c|c|c|c|}
\hline Authors \& Year & No. of Pts & Pt Age (yrs), Sex & Procedure & Engel Class at Last FU & Complications \\
\hline Whiting et al., $2020^{3}$ & 5 & $\begin{array}{c}35, \mathrm{~F} \\
28 \& 30, \mathrm{~F}^{*} \\
21, \mathrm{~F} \\
38, \mathrm{~F} \\
37, \mathrm{~F}\end{array}$ & MRgLITT & $\begin{array}{l}\text { la } \\
\text { la } \\
\text { III } \\
\text { la } \\
\text { II }\end{array}$ & 1 pt w/ quadrantanopia \\
\hline Esquenazi et al., $2014^{9}$ & 2 & $\begin{array}{l}48, \mathrm{~F} \\
25, \mathrm{M}\end{array}$ & MRgLITT/MRgLITT+ATL & I & 1 pt w/ quadrantanopia \\
\hline Clarke et al., $2014^{21}$ & 1 & $22, \mathrm{~F}$ & MRgLITT & 1 & None \\
\hline Thompson et al., $2016^{2}$ & 2 & $\begin{array}{l}32, \mathrm{~F} \\
28, \mathrm{M}\end{array}$ & MRgLITT/MRgLITT+ATL & I & None \\
\hline Barber et al., $2017^{25}$ & 1 & $18, \mathrm{M}$ & MRgLITT & 1 & Delayed IPH resulting in hemiparesis \\
\hline Fayed et al., $2018^{26} \dagger$ & 3 & $\begin{array}{c}16, \mathrm{~F} \\
8, \mathrm{M} \\
6, \mathrm{~F}\end{array}$ & MRgLITT & $\begin{array}{l}\text { I } \\
\text { III } \\
\text { III }\end{array}$ & None \\
\hline Cvetkovska et al., $2018^{27}$ & 1 & $28, \mathrm{M}$ & MRgLITT & 1 & None \\
\hline Current study $\dagger$ & 5 & $\begin{array}{c}16, \mathrm{M} \\
7, \mathrm{~F} \\
5, \mathrm{~F} \\
11, \mathrm{~F} \\
17 \& 18, \mathrm{M}^{*}\end{array}$ & MRgLITT & $\begin{array}{c}\text { II } \\
\text { IV } \\
\text { III } \\
\text { I } \\
\text { III }\end{array}$ & $1 \mathrm{pt} \mathrm{w/} \mathrm{hemianopia}$ \\
\hline
\end{tabular}

ATL = anterior temporal lobectomy; IPH = intraparenchymal hemorrhage.

* The same patient was treated twice.

† Studies in which only pediatric patients were included.

a needle to obtain several core specimens prior to ablation. The obvious risk in this setting is creating blood products that may act as a heat sink for the ablation. Specifically, for PVNH, biopsy is not routinely performed and it was not used for any patients in this report. The radiographic appearance of PVNH was characteristic enough here, but future cases may warrant tissue diagnosis and consideration of the implication on ablation outcomes.

\section{Limitations}

This is a small retrospective case series with limited follow-up. A larger number of children receiving MRgLITT treatment is necessary to draw definitive therapeutic conclusions. In addition, with limited follow-up, there is a risk of overestimating long-term seizure freedom, which is especially of concern in pediatric patients. In comparison to adults treated with similar methods (Table 2), there appears to be less dramatic improvement in seizure outcomes in children, so comparison with children treated with similar methods is necessary.

With limited knowledge about the epileptogenesis of PVNH and other areas of the brain, it is possible that seizure networks may reform over time and require retreatment or additional treatment. With this in mind, longitudinal follow-up is recommended for patients with PVNH who have undergone treatment. Despite these limitations, we believe the findings of the current series help enhance the management of pediatric PVNH-related epilepsy.

\section{Conclusions}

We report a series of children with PVNH-related epi- lepsy treated with MRgLITT and demonstrate the feasibility and safety of the technique. Overall, there was a reduction in seizure burden in these patients, and further study of this technique with comparison to other minimally invasive surgical techniques is warranted.

\section{Acknowledgments}

We thank Kristin Kraus, MSc, for her editorial assistance in preparing this paper.

\section{References}

1. Kwan P, Schachter SC, Brodie MJ. Drug-resistant epilepsy. $N$ Engl J Med. 2011;365(10):919-926.

2. Thompson SA, Kalamangalam GP, Tandon N. Intracranial evaluation and laser ablation for epilepsy with periventricular nodular heterotopia. Seizure. 2016;41:211-216.

3. Whiting AC, Bingaman JR, Catapano JS, Whiting BB, Godzik J, et al. Laser interstitial thermal therapy for epileptogenic periventricular nodular heterotopia. World Neurosurg. 2020;138:e892-e897.

4. Srour M, Rioux MF, Varga C, Lortie A, Major P, et al. The clinical spectrum of nodular heterotopias in children: report of 31 patients. Epilepsia. 2011;52(4):728-737.

5. Aghakhani Y, Kinay D, Gotman J, Soualmi L, Andermann $\mathrm{F}$, et al. The role of periventricular nodular heterotopia in epileptogenesis. Brain. 2005;128(Pt 3):641-651.

6. Raymond AA, Fish DR, Sisodiya SM, Alsanjari N, Stevens JM, Shorvon SD. Abnormalities of gyration, heterotopias, tuberous sclerosis, focal cortical dysplasia, microdysgenesis, dysembryoplastic neuroepithelial tumour and dysgenesis of the archicortex in epilepsy. Clinical, EEG and neuroimaging features in 100 adult patients. Brain. 1995;118(Pt 3):629-660.

7. Park TS, Bourgeois BF, Silbergeld DL, Dodson WE. Subtem- 
poral transparahippocampal amygdalohippocampectomy for surgical treatment of mesial temporal lobe epilepsy. Technical note. J Neurosurg. 1996;85(6):1172-1176.

8. Battaglia G, Arcelli P, Granata T, Selvaggio M, Andermann F, et al. Neuronal migration disorders and epilepsy: a morphological analysis of three surgically treated patients. Epilepsy Res. 1996;26(1):49-58.

9. Esquenazi Y, Kalamangalam GP, Slater JD, Knowlton RC, Friedman E, et al. Stereotactic laser ablation of epileptogenic periventricular nodular heterotopia. Epilepsy Res. 2014; 108(3):547-554.

10. Wyllie E. Wyllie's Treatment of Epilepsy. Lippincott Williams \& Wilkins; 2020.

11. North RY, Raskin JS, Curry DJ. MRI-guided laser interstitial thermal therapy for epilepsy. Neurosurg Clin N Am. 2017; 28(4):545-557.

12. Engel J, ed. Surgical Treatment of the Epilepsies. 2nd ed. Raven Press; 1993.

13. Chabardes S, Abel TJ, Cardinale F, Kahane P. Commentary: understanding stereoelectroencephalography: what's next? Neurosurgery. 2018;82(1):E15-E16.

14. Cossu M, Cardinale F, Colombo N, Mai R, Nobili L, et al. Stereoelectroencephalography in the presurgical evaluation of children with drug-resistant focal epilepsy. J Neurosurg. 2005;103(4)(suppl):333-343.

15. Cossu M, Schiariti M, Francione S, Fuschillo D, Gozzo F, et al. Stereoelectroencephalography in the presurgical evaluation of focal epilepsy in infancy and early childhood. $\mathrm{J} \mathrm{Neu-}$ rosurg Pediatr. 2012;9(3):290-300.

16. Gonzalez-Martinez J, Mullin J, Bulacio J, Gupta A, Enatsu R, et al. Stereoelectroencephalography in children and adolescents with difficult-to-localize refractory focal epilepsy. Neurosurgery. 2014;75(3):258-268.

17. Brandmeir NJ, Sather M. Author response to: Stereotactic accuracy must be as high as possible in stereoelectroencephalography procedures. J Robot Surg. 2018;12(2):387-388.

18. Lefranc M, Le Gars D. Robotic implantation of deep brain stimulation leads, assisted by intra-operative, flat-panel CT. Acta Neurochir (Wien). 2012;154(11):2069-2074.

19. Sarkar A, Dejesus M, Bellamy B, Newton HB, Lo SS. Successful Gamma Knife-based stereotactic radiosurgery treatment for medically intractable heterotopia-based seizure disorder. Clin Neurol Neurosurg. 2011;113(10):934-936.

20. Cossu M, Mirandola L, Tassi L. RF-ablation in periventricular heterotopia-related epilepsy. Epilepsy Res. 2018;142: 121-125.

21. Clarke DF, Tindall K, Lee M, Patel B. Bilateral occipital dysplasia, seizure identification, and ablation: a novel surgical technique. Epileptic Disord. 2014;16(2):238-243.

22. Agari T, Mihara T, Baba K, Kobayashi K, Usui N, et al. Successful treatment of epilepsy by resection of periventricular nodular heterotopia. Acta Med Okayama. 2012;66(6):487-492.

23. Wu C, Sperling MR, Falowski SM, Chitale AV, WernerWasik M, et al. Radiosurgery for the treatment of dominant hemisphere periventricular heterotopia and intractable epilepsy in a series of three patients. Epilepsy Behav Case Rep. 2012;1:1-6.
24. Li LM, Dubeau F, Andermann F, Fish DR, Watson C, et al. Periventricular nodular heterotopia and intractable temporal lobe epilepsy: poor outcome after temporal lobe resection. Ann Neurol. 1997;41(5):662-668.

25. Barber SM, Tomycz L, George T, Clarke DF, Lee M. Delayed intraparenchymal and intraventricular hemorrhage requiring surgical evacuation after MRI-guided laser interstitial thermal therapy for lesional epilepsy. Stereotact Funct Neurosurg. 2017;95(2):73-78.

26. Fayed I, Sacino MF, Gaillard WD, Keating RF, Oluigbo CO. MR-guided laser interstitial thermal therapy for medically refractory lesional epilepsy in pediatric patients: experience and outcomes. Pediatr Neurosurg. 2018;53(5):322-329.

27. Cvetkovska E, Martins WA, Gonzalez-Martinez J, Taylor K, Li J, et al. Heterotopia or overlaying cortex: What about inbetween? Epilepsy Behav Case Rep. 2018;11:4-9.

\section{Disclaimer}

The views expressed herein are those of the author(s) and do not necessarily reflect the official policy or position of the Department of the Navy, Department of Defense, or the U.S. Government.

\section{Disclosures}

Dr. Gonda: consultant for Medtronic and Monteris. Dr. Curry: consultant for and receives non-study-related clinical or research effort from Medtronic.

\section{Author Contributions}

Conception and design: Curry, Ravindra. Acquisition of data: Lee, Patino, Ruggieri. Analysis and interpretation of data: Ravindra. Drafting the article: Curry, Ravindra. Critically revising the article: all authors. Reviewed submitted version of manuscript: all authors. Approved the final version of the manuscript on behalf of all authors: Curry. Administrative/technical/material support: Ikeda.

\section{Supplemental Information Current Affiliations}

Dr. Ravindra: Department of Neurosurgery, Naval Medical Center San Diego, CA.

\section{Correspondence}

Daniel J. Curry: Texas Children's Hospital, Baylor College of Medicine, Houston, TX. djcurry@bcm.edu. 